Musées, Patrimoine et Culture scientifiques et techniques

$162 \mid 2015$

novembre-décembre 2015

\title{
La stratégie numérique du musée de la Civilisation
}

\section{Ana-Laura Baz}

URL : http://journals.openedition.org/ocim/1583

DOI : 10.4000/ocim.1583

ISSN : 2108-646X

\section{Éditeur}

OCIM

Édition imprimée

Date de publication : 1 novembre 2015

Pagination : 25-29

ISSN : 0994-1908

Référence électronique

Ana-Laura Baz, "La stratégie numérique du musée de la Civilisation », La Lettre de I'OCIM [En ligne],

162 | 2015, mis en ligne le 01 novembre 2016, consulté le 19 avril 2019. URL : http://

journals.openedition.org/ocim/1583; DOI : 10.4000/ocim.1583

Ce document a été généré automatiquement le 19 avril 2019

Tous droits réservés 


\title{
La stratégie numérique du musée de la Civilisation
}

\author{
Ana-Laura Baz
}

Séminaire interne pour l'implantation de la stratégie numérique (septembre 2013).

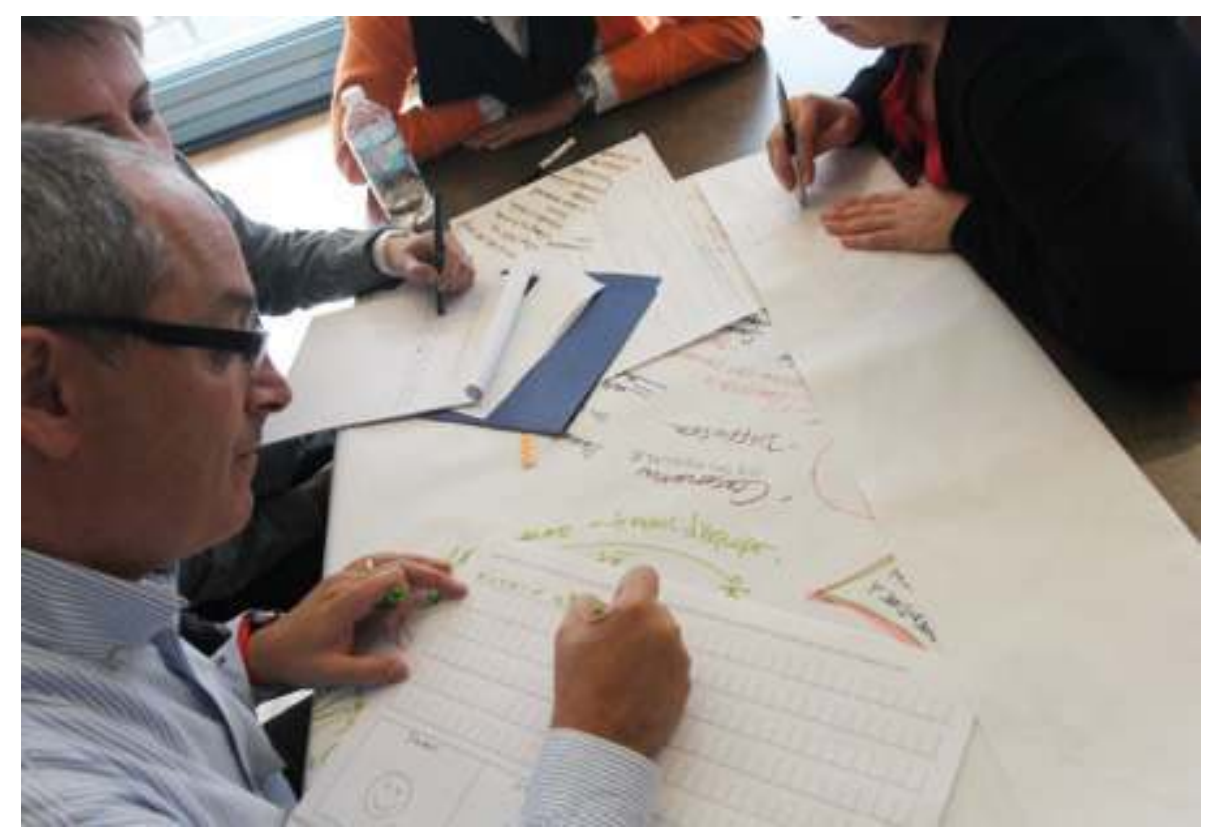

(c) Jean-Sébastien Bouchard

Afin de poursuivre son projet culturel de valorisation et de diffusion de l'héritage culturel québécois et des civilisations du monde, le musée de la Civilisation se devait de tenir compte de l'émergence des nouvelles dynamiques numériques qui animent nos sociétés. Les musées étant des lieux de rencontre avec des idées et des personnes, ces nouvelles réalités numériques contribuent forcément à l'exploration de territoires méconnus de la muséologie. 


\section{Élaborer la stratégie numérique}

2 En juin 2011, le ministère de la Culture du Québec demande à la Société de développement des entreprises culturelles (SODEC) et au Conseil des arts et lettres du Québec (CALQ) de mener une vaste consultation auprès du milieu culturel. L'objectif est d'alimenter le ministère pour l'élaboration d'une stratégie numérique culturelle nationale. Le musée de la Civilisation a participé à ces deux consultations.

3 Au même moment s'amorcent nos propres travaux visant la conception d'une stratégie numérique institutionnelle. Le souhait est de doter le musée de la Civilisation d'une vision du développement numérique qui soit déployée par chacune des unités administratives. C'est également l'occasion de s'interroger sur nos pratiques professionnelles, puisque le numérique bouscule parfois nos savoir-faire. La stratégie doit donc également tenir compte des besoins et enjeux des unités administratives.

4 Voyons maintenant de manière un peu plus concrète quelles ont été les réflexions et orientations porteuses de la stratégie numérique du musée de la Civilisation. Puis, quelques projets réalisés récemment qui illustrent sa mise en application. Pour terminer, nous laisserons la place à quelques constats qui se dégagent déjà de ce déploiement numérique.

\section{S'interroger sur les réalités d'aujourd'hui}

5 Lors de l'élaboration d'une stratégie numérique, il est important de tenir compte des réalités et des comportements observables dans la société, ainsi que des tendances qui se profilent à l'horizon. Voici les réalités et enjeux issus du Web, et des technologies numériques, qui, en 2011, contribuaient déjà aux changements de comportements des citoyens et aux mouvances de nos sociétés :

6 - une vie sociale numérique : les médias sociaux;

7 - la participation et la création citoyennes : des outils pour être, agir et s'exprimer sur le Web ;

8 - la mobilité, l'infonuagique et l'accès au Web en tout temps et en tout lieu : osmose entre la présence physique et le virtuel ;

9 - le besoin d'accéder à du contenu de qualité et de vivre une expérience signifiante : un besoin qui persiste ;

10 - les transactions électroniques autonomes (achats, réservations, formulaires, comptes d'abonnés...) : créer des liens durables avec les publics;

11 - le mouvement des données ouvertes, des communs et la question des droits : passer du copyright aux Creative Commons et à la liberté autoproclamée de photographier partout ;

12 - des fractures numériques : favoriser la démocratisation et l'accessibilité.

13 Ces constats ont constitué les bases alimentant la définition de la stratégie numérique du musée de la Civilisation. C'est en tenant compte de ces réalités et en les arrimant à notre projet culturel que le musée offrira aux citoyens des expériences numériques dynamiques et enrichissantes. 
Lors du séminaire de septembre 2013 , les employés devaient nommer, en équipes, quelle pourrait être l'ambition du musée de la Civilisation en matière de numérique.

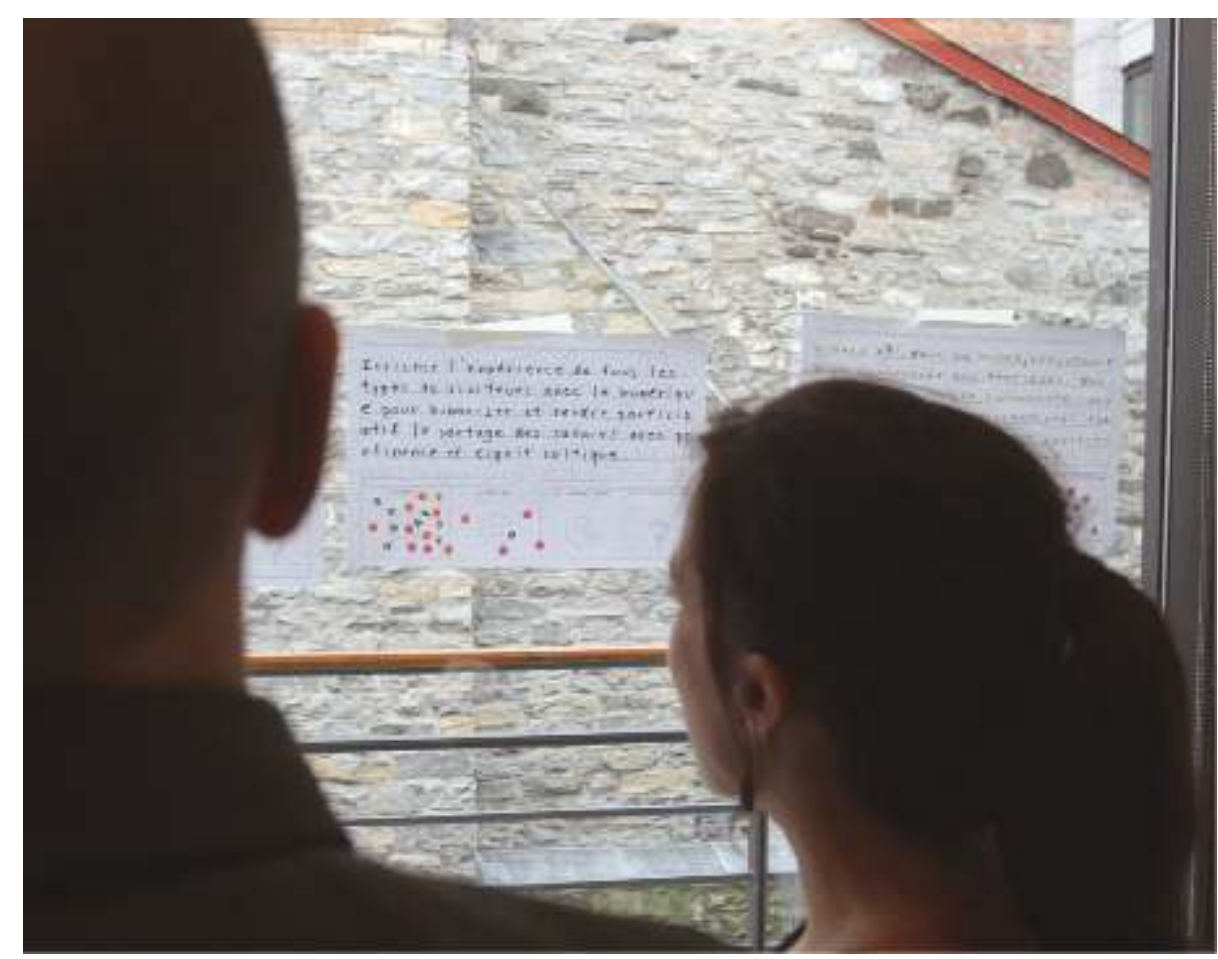

(c) Jean-Sébastien Bouchard

\section{Un objectif général}

14 La stratégie numérique priorise effectivement l'humain, sa pensée, ses intérêts et ses actions. Elle poursuit un objectif de connaissance, de rencontre et de liaison. Il s'agit de permettre aux citoyens l'accès, l'interaction et le partage des contenus et des informations, avec d'autres visiteurs, des professionnels du musée et des spécialistes.

La portée de cet objectif s'étend bien au-delà des visiteurs qui franchissent les portes du musée. En raison du caractère dématérialisé, ubiquitaire et asynchrone du Web, c'est bel et bien l'ensemble des citoyens qui sont visés, tant au Québec qu'ailleurs dans le monde.

\section{La stratégie numérique du musée de la Civilisation}

Le musée s'est ainsi doté d'une stratégie numérique qui s'articule autour de cinq orientations, elles-mêmes déployées en divers axes :

- enrichir l'offre de contenus diffusés de façon numérique : site Web, collections en ligne, expositions virtuelles, édition numérique ;

18 - développer une expérience numérique en continuité avec celle in situ: applications mobiles, médias sociaux, affichage et bornes dans le musée, déployant des actions sur le Web et les médias sociaux;

19 - favoriser la participation citoyenne au sein de projets liés aux expositions, à la médiation, aux expositions virtuelles, ou auprès de publics spécialisés ; 
20 modes de vie liés au transport à cheval. Elles suscitent une certaine nostalgie, que nous avons souhaité transformer en inspiration créatrice. Quatre voitures sont accompagnées d'artefacts évoquant une ambiance poétique : chapeau-melon et canari, peluche oubliée, soirée hivernale et pique-nique du dimanche. Les visiteurs sont alors invités à créer, sur des iPad, une courte histoire de fiction en 140 caractères : la twittérature.

Manger ensemble ! explore le patrimoine alimentaire par ses provenances régionales et ethniques, sa capacité à forger des sentiments d'appartenance, ses modes de transmission et son ancrage fort dans le territoire.

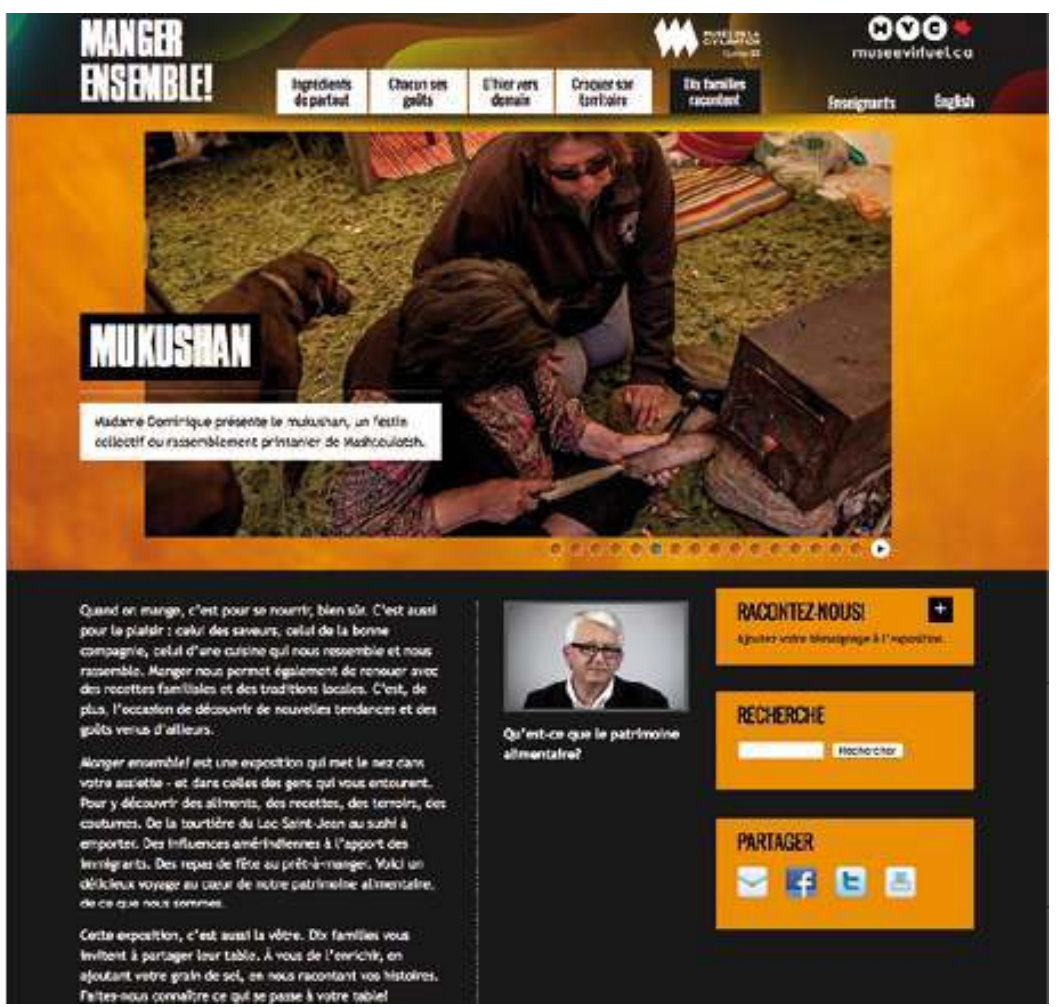

(c) Musée de la Civilisation 


\section{Manger ensemble !}

24

Cette exposition virtuelle participative propose un voyage au cœur de notre patrimoine alimentaire (mangerensemble.mcq.org). Des dizaines d'entretiens et de témoignages recueillis auprès de dix familles d'origines variées permettent d'explorer la diversité du patrimoine alimentaire au Québec. Une invitation est ensuite lancée aux citoyens afin qu'ils partagent leurs propres témoignages par le biais d'un texte, d'une photo ou d'une vidéo.

Publications numériques THEMA et Variations

Ces deux publications scientifiques, éditées par l'équipe de la recherche du musée de la Civilisation, sont exclusivement numériques (thema.mcq.org et www.mcq.org/variations) et assurent la diffusion de savoirs scientifiques auprès de la communauté muséale. THEMA est une revue internationale, interdisciplinaire et multilingue qui explore les rapports entre les sociétés et les musées. Variations diffuse plutôt les résultats de recherches produites dans le cadre de projets institutionnels par des membres de l'équipe du musée de la Civilisation ou par des chercheurs externes.

\section{Les applications mobiles}

Le musée a produit neuf applications mobiles depuis novembre 2009. Ces applications ont visé des publics variés : adultes, touristes, personnes sourdes utilisant la langue des signes québécoise et enfants. Elles proposaient un parcours complémentaire à la visite de l'exposition (visite commentée, jeu, contenus vidéo inédits, interaction avec des contenus de l'exposition) ou une expérience de création.

8 Il a été constaté que l'utilisation d'une application mobile prolonge considérablement l'expérience de visite. L'évaluation de l'application Copyright humain (visite commentée destinée aux adultes) a permis de découvrir que $75 \%$ des personnes visitant l'exposition sans iPod exploraient l'exposition durant moins de 30 minutes. En revanche, $50 \%$ des personnes utilisant un iPod effectuaient une visite de plus d'une heure ${ }^{1}$. Le même constat a été fait pour l'application Paris Belle Époque avec Titi, offrant une visite commentée aux enfants de 6 à 10 ans $^{2}$.

Cet accroissement de la durée de visite s'accompagne d'un nouvel intérêt envers les constituantes de l'exposition: "Certains comportements sont induits par l'application mobile, notamment l'attention portée aux détails des objets et la lecture des vignettes et des textes. Plusieurs utilisateurs ont mentionné que le jeu les avait incités à lire plus que d'habitude, que ce soit pour résoudre les épreuves ou tout simplement par intérêt pour un objet qu'ils auraient été moins portés à regarder en contexte de visite libre $»^{3}$.

\section{Médias sociaux}

La perspective selon laquelle seront développés les médias sociaux s'approche plus de la médiation que de la mise en marché. Notre souhait est de favoriser l'engagement des citoyens envers le musée en mettant en valeur nos contenus et nos collections. 
Du $1^{\text {er }}$ décembre 2014 au 4 janvier 2015, le musée a publié quotidiennement sur les médias sociaux une photographie d'un artefact, d'une activité ou un concours. Ces publications ont été visionnées près de 200000 fois.
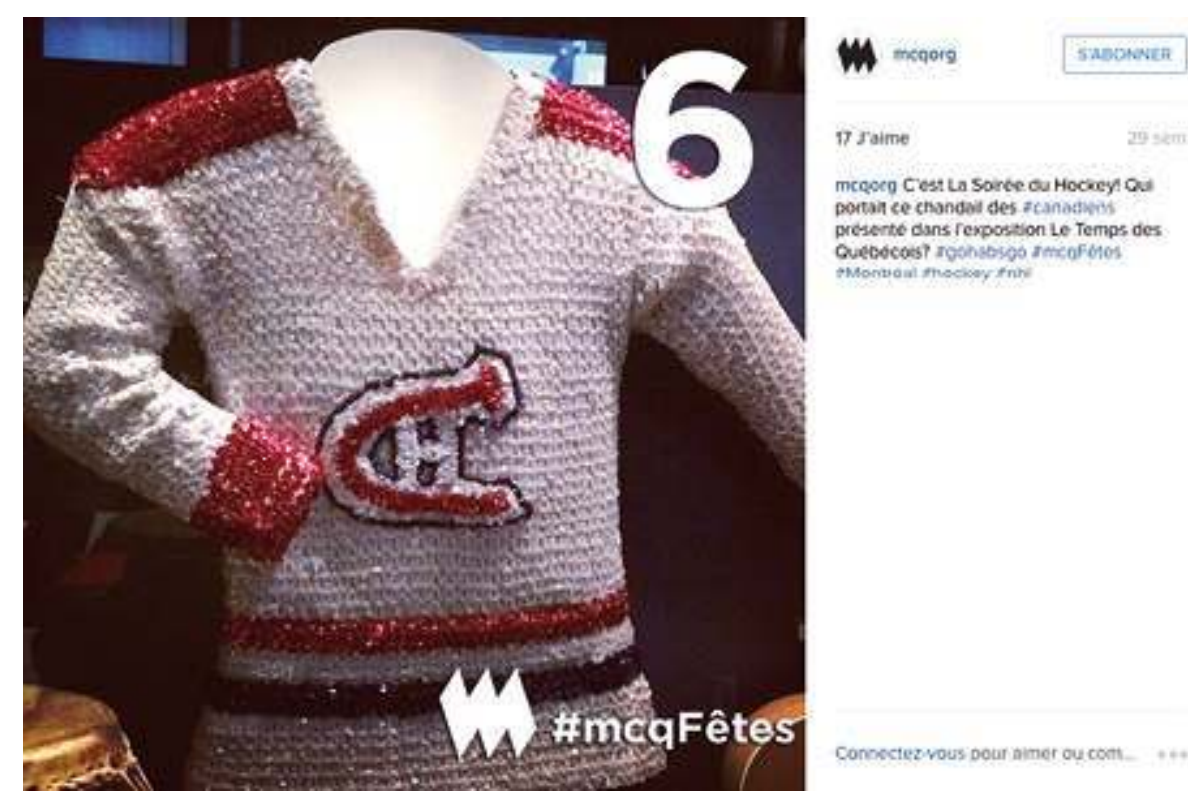

(c) Musée de la Civilisation

\section{Les constats}

\section{Expérimenter}

42 Le musée de la Civilisation s'est donné la liberté d'essayer plusieurs approches, plusieurs technologies, plusieurs types d'expériences numériques : plus de 60 projets depuis 2008. Ces réalisations nous on permis de travailler avec des créateurs et des réalisateurs qui, à leur tour, nous ont fait découvrir de nouvelles voies où porter nos projets. 

définir nos projets, à les cibler, à les clarifier, à les intégrer plus étroitement au projet culturel du musée de la Civilisation. Cette somme d'expériences numériques constitue nos fondations pour poursuivre l'aventure.

\section{Prétester, analyser, évaluer}

Nous avons prétesté nos projets à l'interne, avec des visiteurs et avec des citoyens en dehors du musée. Nous avons accepté que des ajustements soient parfois nécessaires.

Nous avons analysé les statistiques d'utilisation et de visionnement de nos réalisations pour les expositions virtuelles, le site Web, Facebook, Twitter, YouTube... Nous avons observé où celà fonctionnait, à quel moment, avec quel type d'utilisateur. Nous avons ainsi mieux compris les spécificités de chaque espace numérique. Nous avons également évalué des projets après leur lancement, en particulier les applications mobiles, afin de recueillir les impressions des visiteurs qu'ils les aient utilisées ou non.

Chaque prétest, analyse et évaluation nous a outillés dans l'amélioration de notre pratique professionnelle et pour la planification des projets à venir.

\section{Plus de contenus en ligne, des résultats?}

Nous le savons, le Web est un amoncellement de tout et de n'importe quoi. Surtout de n'importe quoi. Pensons aux vidéos sur YouTube, aux statuts Facebook qui ne devraient pas être publics, aux banalités sur Twitter et aux photographies gênantes. Plusieurs se sont également plaints de l'omniprésence de l'anglais.

Les établissements culturels et de savoir font maintenant le constat qu'ils doivent diffuser des contenus rigoureux, originaux, créatifs et de qualité sur le Web et dans les médias sociaux. Le numérique s'ajoute alors à l'éventail des approches, moyens et stratégies de diffusion et de médiation muséale.

Après toutes ces expériences en diffusion de contenus textuels, vidéo et photographiques, notre culture est-elle consultée sur le Web ? Voyage-t-elle partout dans le monde ? Oui. À titre d'exemple, le live tweet des conférences scientifiques du symposium en évaluation (1 er et 2 décembre 2014) a été visionné près de 54000 fois. Durant l'année 2013, l'exposition virtuelle Place-Royale, d'aujourd'hui à hier a été consultée dans presque tous les pays du monde. Ces chiffres sont séduisants. Mais que veulent-ils dire? Les textes ont-ils été lus ? Les messages ont-ils un effet? Les gens ont-ils appris quelque chose? Leurs manières d'être, de penser et d'agir ont-elles changé ?

Nous n'avons pas toutes les réponses à ces questions. Les moyens pour évaluer ces retombées sont difficiles à mettre en place. Ce constat ne discrédite pas les chiffres. Il ne fait que confirmer qu'il y a encore beaucoup à faire. Des recherches devront être menées afin que nous puissions trouver des manières d'aller au-delà des chiffres pour mieux comprendre les comportements des internautes.

La Lettre de l'OCIM, 162 | 2015 
Laboratoire d'animation de l'exposition Image x image. Le cinéma d'animation à l'ONF.

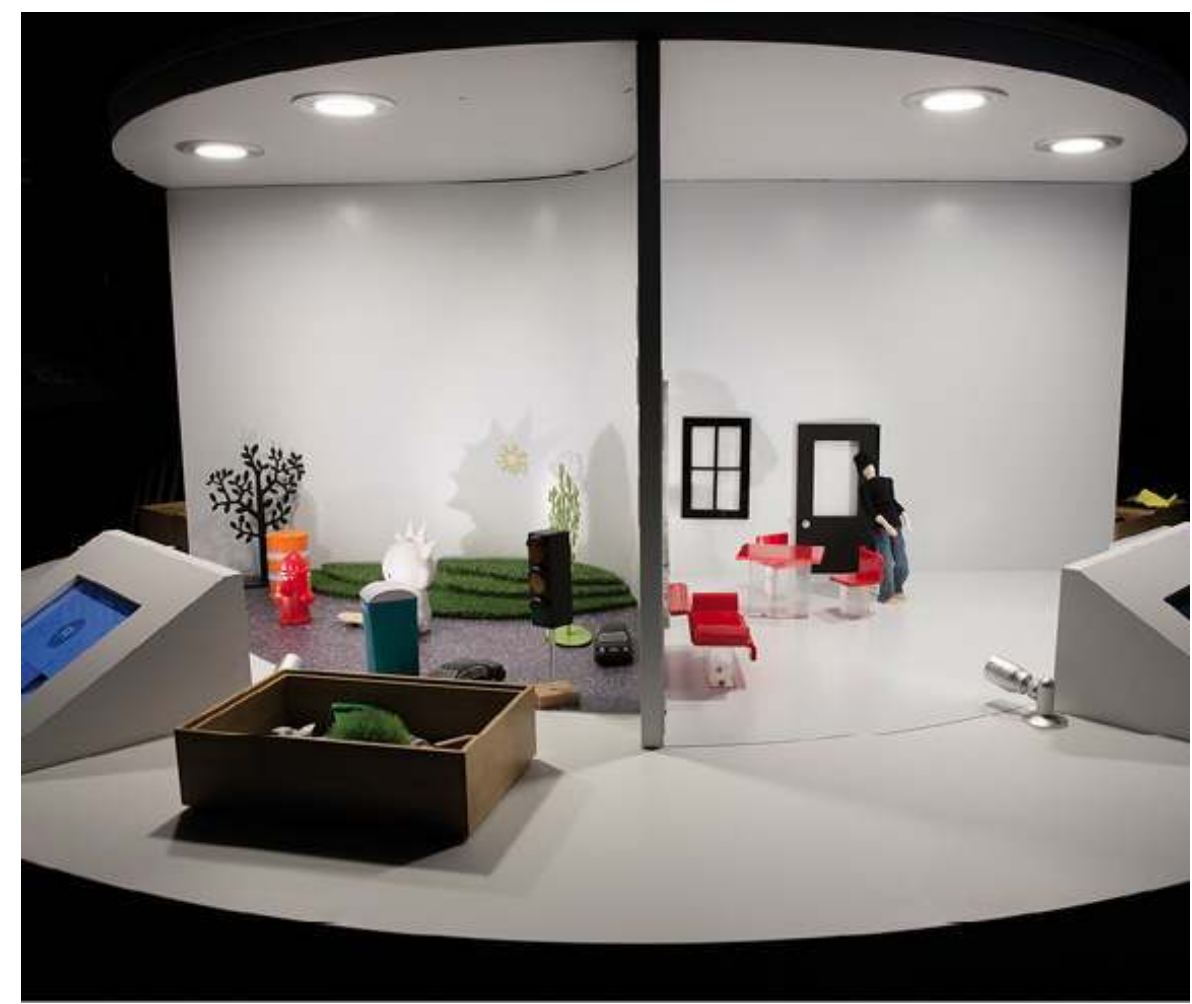

(c) Musée de la Civilisation/Jessy Bernier, Perspective Photo

\section{Et la participation citoyenne, ça fonctionne?}

51 Oui. Et non. En fait, il n'y a rien d'automatique. Parce qu'il y a différents types de participation citoyenne. Parce que certains sujets sont plus attirants que d'autres. Parce que certains projets, bien qu'intéressants, passent sous le radar : les gens n'en entendent pas parler. Parfois, parce que nous ne mettons pas les efforts pour les publiciser. Parce que parfois nous ciblons un public et présumons qu'il sera au rendez-vous, mais qu'il n'y est tout simplement pas.

52 Voici quelques exemples d'expériences de participation citoyenne numérique qui illustrent cette diversité de situations :

53 - Webdiffusion des rencontres thématiques de panélistes Participe présent (2010-2011) : en plus de la participation en salle par l'échange avec les spécialistes invités, les citoyens pouvaient visionner en direct la rencontre par webdiffusion, et une séance de clavardage était animée par le musée. Une moyenne de 80 personnes ont visionné la webdiffusion, selon le sujet de la rencontre, mais les questions et échanges de clavardage se comptent sur les doigts de la main. Très peu de publicité avait été déployée, et la webdiffusion était encore méconnue (nous étions loin des technologies Périscope et de Meerkat) ;

54 - Laboratoire de création de films d'animation dans l'exposition Image x image. Le cinéma d'animation à l'ONF : six stations offrent l'application mobile Animation iXi, afin que les gens expérimentent différentes techniques de réalisation de films d'animation. Près de 8000 vidéos ont été créées par le public, et elles ont été visionnées 90000 fois ; 
- Museomix Québec 2013 (7, 8 et 9 novembre 2013) : au-delà des 250 personnes qui ont organisé cet événement où la muséologie rencontre le numérique et qui y ont participé, c'est plutôt vers ses retombées qu'il faut se tourner pour en mesurer le succès. Il s'agissait d'explorer de nouvelles approches muséales (le numérique), d'imaginer de nouvelles manières de penser la place du citoyen-visiteur au musée, d'intégrer la «culture du faire » et la «culture numérique » au sein de l'équipe du musée et d'ouvrir le musée à de nouvelles formes de collaboration (communautés).

Numérisation 3D d'une sculpture inuit lors de l'événement Museomix Québec 2013 au musée de la Civilisation.

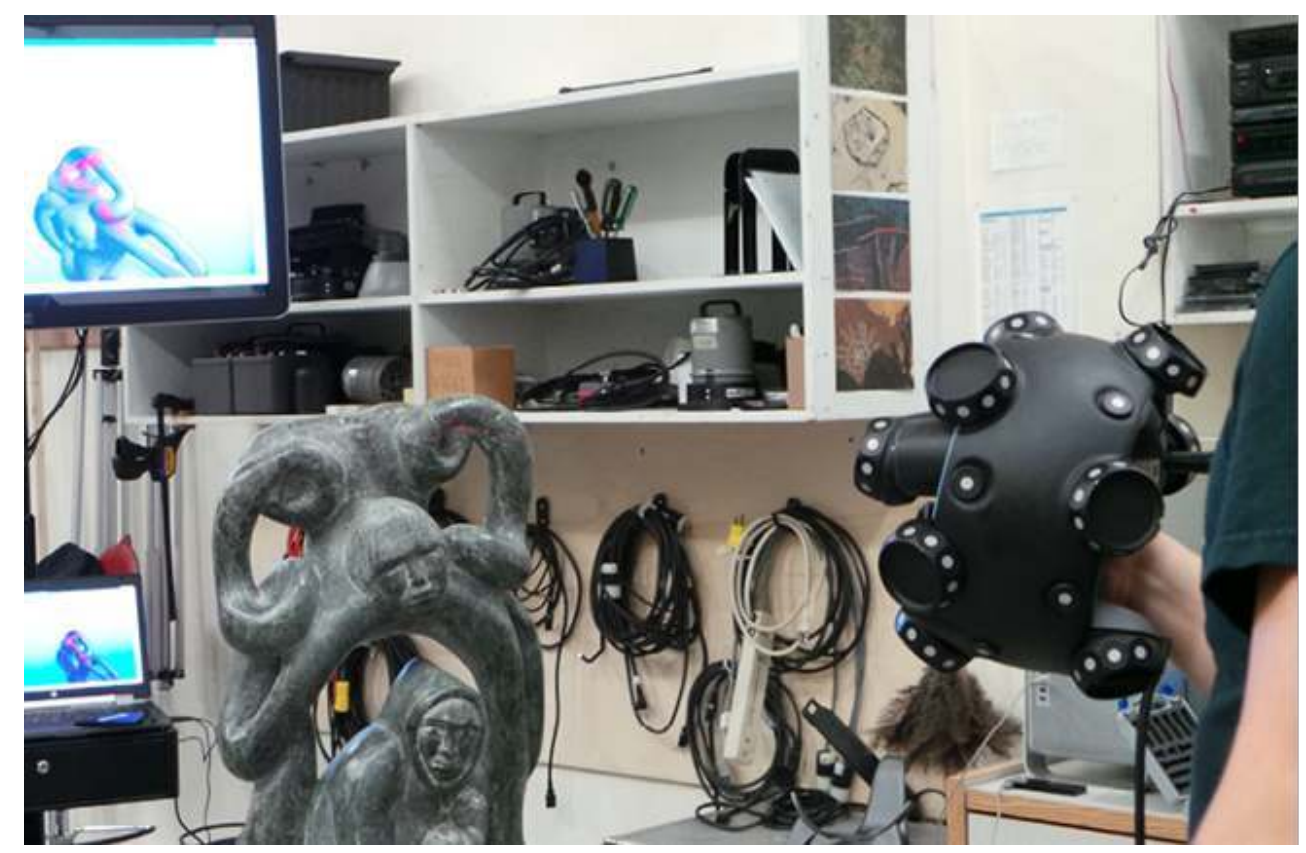

(c) Museomix Québec

Pour réussir la participation citoyenne, les efforts doivent véritablement débuter dès que le projet est lancé : il faut en parler publiquement et élargir progressivement le groupe des personnes impliquées. Or il est trop souvent présumé que notre projet deviendra viral par sa simple présence en ligne et qu'il n'est pas nécessaire de déployer des efforts pour la promotion et les relations de presse des projets numériques. C'est faux. Rares sont les contenus numériques qui se font connaître sans efforts de mise en marché. De plus, une fois que le projet de participation citoyenne est lancé, il faut effectuer la modération des contributions du public et poursuivre les efforts de promotion. Ces démarches demandent du temps, des personnes responsables et des investissements qui sont trop souvent négligés.

\section{Miser sur la culture numérique}

57 Le numérique englobe dorénavant plus que la technologie et s'oriente vers ce qui est généralement appelé la culture numérique. Une culture du partage, de la réutilisation, de la dissémination, de la cocréation et de l'autonomie d'action numérique des citoyens. Ces principes peuvent s'ancrer dans les musées, notamment par la question de la photographie citoyenne en exposition, l'octroi de licences de libre utilisation des 
contenus numériques et par les outils de transactions électroniques (réservations, achats, abonnements...). D'autres déploiements voient le jour au sein des équipes de travail, comme les logiciels de travail collaboratif et l'infonuagique.

Bien que le numérique soit dématérialisé (virtuel), il n'en demeure pas moins réel. Il n'y a pas une « vraie vie en présentiel » et une « fausse vie virtuelle » : il n'y a qu'une seule vie où le virtuel et le physique se complètent et se nourrissent mutuellement. Cette culture ne s'apprend pas sur les bancs d'école ou dans les livres. La seule façon de la développer, c'est de la vivre.

\section{NOTES}

1. Daignault, L., Cousson, C. et Dupret, T. Copyright humain. La visite commentée avec un iPod touch. Usages et réactions. Service de la recherche et de l'évaluation. Musée de la Civilisation, novembre 2010.

2. Daignault, L. et Corriveau, M.-É. L'application mobile Paris Belle Époque avec Titi. Retour sur l'expérience vécue en famille. Service de la médiation culturelle et éducative. Musée de la Civilisation, décembre 2013.

3. Daignault, L. et Brouillette, A. L'application mobile "Deviens Samouraï". Quel impact sur l'expérience de visite des familles? Secrétariat général. Musée de la Civilisation, avril 2013.

\section{RÉSUMÉS}

Après une description des orientations déployées dans la mise en œuvre de la stratégie numérique du musée de la Civilisation à Québec, l'auteur dresse un premier bilan de l'opération, de ses succès et de ses échecs, constatant que beaucoup reste à faire pour mesurer les retombées de cette stratégie sur la participation citoyenne et le comportement des internautes.

\section{INDEX}

Mots-clés : stratégie numérique, musée de la civilisation, Québec

\section{AUTEUR}

\section{ANA-LAURA BAZ}

chargée de projets numériques au musée de la Civilisation albaz@mcq.org 